\title{
An overview of carbapenem, its resistance and therapeutic options for infections caused by carbapenem resistant bacteria
}

\author{
Hari P. Nepal ${ }^{1 *}$, Rama Paudel ${ }^{2}$
}

\author{
${ }^{1}$ School of Medicine, ${ }^{2}$ School of Biomedical Sciences, Trinity Medical Sciences University, Kingstown, St. Vincent \\ and the Grenadines
}

Received: 23 June 2020

Accepted: 31 July 2020

\author{
*Correspondence: \\ Dr. Hari P. Nepal, \\ Email:drharinepal@gmail.com
}

Copyright: $(\odot$ the author(s), publisher and licensee Medip Academy. This is an open-access article distributed under the terms of the Creative Commons Attribution Non-Commercial License, which permits unrestricted non-commercial use, distribution, and reproduction in any medium, provided the original work is properly cited.

\begin{abstract}
Carbapenems are beta-lactam drugs that have broadest spectrum of activity. They are commonly used as the drugs of last resort to treat complicated bacterial infections. They bind to penicillin binding proteins (PBPs) and inhibit cell wall synthesis in bacteria. Important members that are in clinical use include doripenem, ertapenem, imipenem, and meropenem. Unlike other members, imipenem is hydrolyzed significantly by renal dehydropeptidase; therefore, it is administered together with an inhibitor of renal dehydropeptidase, cilastatin. Carbapenems are usually administered intravenously due to their low oral bioavailability. Most common side effects of these drugs include nausea, vomiting, diarrhea, skin rashes, and reactions at the infusion sites. Increasing resistance to these antibiotics is being reported throughout the world and is posing a threat to public health. Primary mechanisms of carbapenem resistance include expulsion of drug and inactivation of the drug by production of carbapenemases which may not only hydrolyze carbapenem, but also cephalosporin, penicillin, and aztreonam. Resistance especially among Gram negative bacteria is of much concern since there are only limited therapeutic options available for infections caused by carbapenem resistant Gram-negative bacterial pathogens. Commonly used drugs to treat such infections include polymyxins, fosfomycin and tigecycline.
\end{abstract}

Keywords: Carbapenems, Drugs of last resort, Resistance, Therapeutic options

\section{INTRODUCTION}

Carbapenems are the important drugs in our antibiotic armamentarium. Of the various beta-lactams, carbapenems exhibit the broadest spectrum of activity and greatest potency against Gram-positive and Gramnegative bacteria. ${ }^{1}$ They are often regarded as "lastline agents" or "antibiotics of last resort" in treatment of serious infections. ${ }^{2}$ However, emergence of carbapenem resistant pathogens such as carbapenem resistant Enterobacteriaceae (CRE), Acinetobacter baumannii and Pseudomonas aeruginosa in infections such as hospital acquired infections has not only seriously threatened this class of lifesaving drugs but also have posed a significant threat to public health. ${ }^{1,3}$ These resistant pathogens are difficult to treat and are associated with high mortality. ${ }^{3}$

The purpose of this article is to review the important pharmacological characteristics of carbapenem and its resistance as well as to find out the options available for treatment of infections caused by carbapenem resistant bacterial pathogens.

A literature search was done using Medline database, Google database and online journals. The search was limited to publications in the English language. The search terms 'carbapenem', 'pharmacology of carbapenem', 'carbapenem resistance' and 'therapeutic/treatment options for infections caused by 
carbapenem resistant pathogens' were used. Additional information was obtained from standard book and the websites of agencies such as Centers for Disease Control and Prevention (CDC), World Health Organization (WHO) and European centre for disease prevention and control.

\section{STRUCTURE OF CARBAPENEM}

Structurally, carbapenems are similar to the penicillins (penams), but the sulfur atom in position 1 of the structure of penicillin has been replaced with a carbon atom. ${ }^{1}$ Thus, their bicyclic core consists of a beta-lactam ring fused to a pyrroline ring (Figure 1). Combination of these two rings confers exceptional stability against most beta-lactamases (enzymes that inactivate beta-lactams) including ampicillin and carbenicillin (Amp C) and the extended spectrum beta-lactamases (ESBLs). ${ }^{4}$

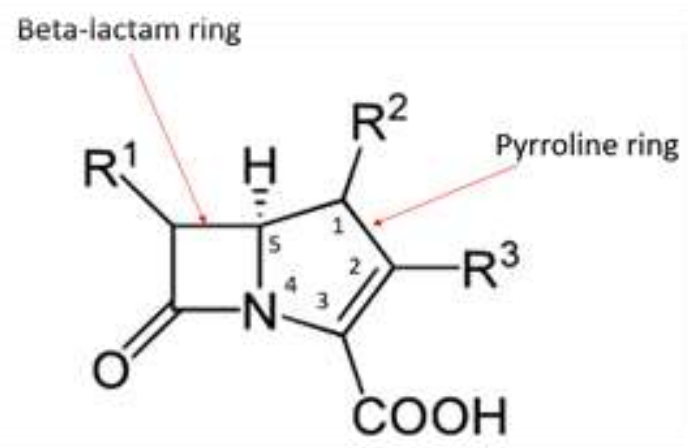

Figure 1: Structure of carbapenem.

\section{MECHANISM OF ACTION}

Carbapenems bind to penicillin binding proteins (PBPs) which are enzymes (i.e., transpeptidases, transglycolases, and carboxypeptidases) that catalyze the formation of peptidoglycan in the cell wall of bacteria. ${ }^{1}$ They permanently acylate the PBPs, inhibit the cell wall synthesis and kill the bacterial cells. ${ }^{1}$

\section{MEMBERS APPROVED FOR USE IN UNITED STATES}

Of its various members, doripenem, ertapenem, imipenem, and meropenem are approved for use in the United States. $^{5}$

\section{SPECTRUM OF ACTIVITY AND RESPONSE TO DEHYDROPEPTIDASE 5}

Imipenem is the first member of carbapenem available for the treatment of complex bacterial infections. It has a wide spectrum with good activity against many Gramnegative rods, including $P$. aeruginosa, Gram positive organisms, and anaerobes. It is resistant to most betalactamases but not carbapenemases or metallo beta- lactamases. Common bacteria resistant to this drug include Enterococcus faecium, methicillin-resistant strains of Staphylococci, Clostridium difficile, Burkholderia cepacia, and Stenotrophomonas maltophilia. This drug is inactivated by dehydropeptidases in renal tubules, resulting in low urinary concentrations. Consequently, it is administered together with an inhibitor of renal dehydropeptidase, cilastatin, for clinical use.

Doripenem and meropenem are similar to imipenem but are more effective Gram-negative aerobes and slightly less effective against Gram positive bacteria. These drugs are not significantly hydrolyzed by renal dehydropeptidase and do not require an inhibitor.

Ertapenem has lesser activity than the other carbapenems against $P$. aeruginosa and Acinetobacter species. It is not hydrolyzed by renal dehydropeptidase.

\section{PHARMACOKINETICS}

All clinically useful carbapenems have low oral bioavailability and are poorly absorbed through gastrointestinal tract. ${ }^{1}$ Therefore, they must be administered intravenously but imipenem-cilastatin and ertapenem can also be delivered intramuscularly. ${ }^{6}$ They penetrate body tissues and fluids well, including the cerebrospinal fluid. ${ }^{5}$

All carbapenems similar to other beta-lactams are eliminated predominately by renal excretion and the dose must be adjusted in patients with renal insufficiency. ${ }^{5}$ Carbapenems exhibit unique pharmacological properties and are typically used to treat complicated bacterial infections. ${ }^{5}$

\section{ADVERSE EFFECTS}

Nausea, vomiting, diarrhea, skin rashes, and reactions at the infusion sites are the most common adverse effects of carbapenems which tend to be more common with imipenem. ${ }^{5}$ In patients with renal failure, excessive levels of imipenem may lead to seizures. Meropenem, doripenem, and ertapenem are much less likely to cause seizures than imipenem. ${ }^{5}$

Nephrotoxicity, neurotoxicity, and immunomodulation have also been reported with the use of carbapenems, and thus patient's underlying conditions and predisposing factors should be considered when administering any carbapenem. ${ }^{1}$ In addition, the use of carbapenems has also been found to alter the intestinal microflora and select for carbapenem resistant isolates. ${ }^{1}$

\section{RESISTANCE}

Literatures have shown that resistance to carbapenems is increasing throughout the world. ${ }^{7-9}$ The resistance is considered as one of major health problems worldwide 
and limits the choice of selected antibiotic therapies to treat bacterial infections.

\section{Mechanism of resistance}

Bacteria exhibit two types of resistance to carbapenems: intrinsic and acquired. ${ }^{4}$ Bacteria such as Stenotrophomonas maltophilia which possesses endogenous metallo-beta-lactamase (MBL) L1 are intrinsically resistant. ${ }^{10}$ However, this type of resistance is not common among clinically important bacteria and for most of them carbapenem resistance is acquired by mutational events or gene acquisition via horizontal gene transfer. $^{4}$ The primary mechanisms of carbapenem resistance mediated by gene acquisition include expulsion of drug and inactivation of the drug by production of carbapenemases. The important mechanisms are outlined below:

\section{Mutation}

Mutation of penicillin binding proteins alters the target and carbapenem is unable to bind to them whereas mutation in outer membrane porin proteins (through which drugs enter into bacterial cell) of gram-negative bacteria prevents carbapenems reaching their PBPs by diminishing the permeability of their outer membrane. ${ }^{4}$

\section{Expulsion of drugs}

Expulsion of carbapenems out of the periplasmic space commonly occurs through a tripartite efflux pump systems composed of a protein transporter of the cytoplasmic membrane, a periplasmic connective protein and an outer membrane porin. ${ }^{4}$ The overexpression of efflux pumps is commonly associated with multidrug resistance (MDR), since quinolones, penicillins, cephalosporins and aminoglycosides are common efflux pump substrates. ${ }^{4}$

\section{Inactivation by enzymes}

Carbapenemases produced by bacteria can hydrolyze carbapenems and render the drug ineffective. They are considered the most potent beta-lactamases. ${ }^{4,11}$ In general, they also hydrolyze other beta-lactam drugs such as cephalosporins, penicillin, and aztreonam. They belong to different Ambler classes (Table 1). ${ }^{11}$

In terms of carbapenem hydrolysis and geographical spread, the most effective carbapenemases are KPC, VIM, IMP, NDM and OXA-48 types. ${ }^{4}$

Carbapenemases coding genes are commonly located on mobile genetic elements and have the potential to be widespread all over the world. ${ }^{11}$ They were originally described in gram-positive bacteria such as Bacillus cereus and Bacillus licheniformis. ${ }^{11}$ However, they are now reported commonly on Gram-negative bacteria such as members of Enterobacteriaceae, $P$. aeruginosa and Acinetobacter species. ${ }^{11}$

Distinct mechanisms of resistance have been observed in Gram-positive cocci and Gram-negative rods. ${ }^{1}$ In Grampositive cocci, carbapenem resistance is typically the result of alteration of $\mathrm{PBP}$, whereas production of carbapenemases, expression efflux pumps, mutation in porin and alterations in PBP are associated with the resistance in Gram-negative rods with the first one being the primary mechanism. ${ }^{1,11}$

Table 1: Types of carbapenemases.

\begin{tabular}{|c|c|}
\hline $\begin{array}{l}\text { Ambler } \\
\text { carbapenemase group }\end{array}$ & Enzyme family \\
\hline \multirow{5}{*}{ Group A } & $\begin{array}{l}\text { Not metalloenzyme } \\
\text { carbapenemase (NmcA) }\end{array}$ \\
\hline & $\begin{array}{l}\text { Serratia marcescens enzyme } \\
(\mathrm{SME})\end{array}$ \\
\hline & $\begin{array}{l}\text { Imipenem-hydrolyzing beta- } \\
\text { lactamase (IMI) }\end{array}$ \\
\hline & $\begin{array}{l}\text { Klebsiella pneumoniae } \\
\text { carbapenemase (KPC) }\end{array}$ \\
\hline & $\begin{array}{l}\text { Guiana extended spectrum } \\
\text { (GES) }\end{array}$ \\
\hline \multirow{3}{*}{ Group B } & Imipenemase (IMP) \\
\hline & $\begin{array}{l}\text { Verona-integron-encoded } \\
\text { metallo-beta-lactamase } \\
\text { (VIM) }\end{array}$ \\
\hline & $\begin{array}{l}\text { New Delhi metallo-beta- } \\
\text { lactamase (NDM) }\end{array}$ \\
\hline \multirow{3}{*}{ Group C (emerging) } & AmpC type (ACT) \\
\hline & Cephamycinase (CMY) \\
\hline & $\begin{array}{l}\text { Acinetobacter-derived } \\
\text { cephalosporinase (ADC) }\end{array}$ \\
\hline
\end{tabular}

\section{RISK GROUPS FOR CARBAPENEM RESISTANT BACTERIAL INFECTIONS}

As outlined by $\mathrm{CDC}$, the people whose care requires devices like ventilators (breathing machines), urinary (bladder) catheters, or intravenous (vein) catheters, patients who are taking long courses of certain antibiotics, and patients with weakened immune systems are at high risk for carbapenem resistant bacterial infections, particularly infections with CRE. ${ }^{12}$

\section{EPIDEMIOLOGY OF CARBAPENEM RESISTANT BACTERIA}

Rapid spread of carbapenem resistant bacteria worldwide is an important concern. ${ }^{13}$ However, exact global prevalence of all these bacteria remains unclear. Of these bacteria, carbapenem-resistant $P$. aeruginosa (CRPA), $A$. baumannii (CRAB) and Enterobacteriaceae (CRE) are the important pathogens causing hospital acquired infections (HAIs) and are prioritized by the WHO as 
critical pathogens that require urgent drug research and the development. ${ }^{13,14}$

A study done over 20 years (1997-2016) from the SENTRY anti-microbial surveillance program which included carbapenem resistance rates ( $\mathrm{n}=52022$ isolates) globally (except Africa and the Middle-East) indicated that carbapenem resistance in P. aeruginosa was $17.4 \%$ (as per the susceptibility testing determined by CLSI guidelines). ${ }^{15}$ European centre for disease prevention and control in 2017 also reported high rates $(25-60 \%)$ of carbapenem resistance in $P$. aeruginosa in Southern and Eastern Europe. ${ }^{16}$

Globally carbapenem resistance was detected in 40-70\% of the isolates of $A$. baumanii responsible for ICUacquired infections. ${ }^{17}$ In the United States, the resistance rates among $A$. baumanii in central line-associated blood stream infection and catheter-associated urinary tract infections were reported as $47 \%$ and $64 \%$, respectively. ${ }^{18,19}$ In Europe, it was found to be $33.4 \%$ among the isolates causing blood stream infection. ${ }^{16}$

The global epidemiology of CRE is yet to be known. ${ }^{13} \mathrm{~A}$ prospective, multinational study on carbapenemaseproducing Enterobacteriaceae (CPE) has reported infection prevalence of CPE to be 1.3 per 10000 hospital admissions. $^{20}$ In the United States, a systemic review highlighted that that incidence of CRE was found to be 0.3-2.93 infections per 100000 person-years. ${ }^{21}$ Over $10 \%$ of $K$. pneumoniae, a member of Enterobacteriaceae, that causes health care associated infections in US hospitals are currently found to be carbapenem resistant. ${ }^{22}$ The prevalence of CRE infections in the community remains unclear, but a review published in 2017 reported it in the range of 0.04 to $29.5 \% .^{23}$

\section{HUMAN-RELATED FACTORS CONTRIBUTING TO EMERGENCE AND SPREAD OF CARBAPENEM RESISTANCE}

The major human related factors that contribute to emergence and spread of carbapenem resistance are reported as: (a) immoderate antibiotic prescription combined with the uncontrolled public access to antibiotics in many countries with poor sales regulations (b) the lack of infection control measures in healthcare facilities once carbapenem resistance has emerged and (c) the use of subtherapeutic doses of antibiotics for the promotion of animal growth in the agricultural sector. ${ }^{4}$

\section{PREVENTION AND CONTROL OF INFECTIONS WITH CARBAPENEM RESISTANT PATHOGENS}

WHO recommends the following measures for prevention and control of infections associated with carbapenem resistant pathogens, particularly CRE. ${ }^{3}$

\section{Recommendation 1}

Multimodal IPC strategies should be implemented to prevent and control CRE infection or colonization and that these should consist of at least the following: hand hygiene, surveillance, contact precautions, patient isolation (single room isolation or co-horting) and environmental cleaning.

\section{Recommendation 2}

Hand hygiene best practices according to the WHO guidelines on hand hygiene in health care should be implemented.

\section{Recommendation 3}

Surveillance of CRE infection (s) should be performed; and surveillance cultures for asymptomatic CRE colonization should also be performed, guided by local epidemiology and risk assessment. Populations to be considered for such surveillance include patients with previous CRE colonization, patient contacts of CRE colonized or infected patients and patients with a history of recent hospitalization in endemic CRE settings.

\section{Recommendation 4}

Contact precautions should be implemented when providing care for patients colonized or infected with CRE.

\section{Recommendation 5}

Patients colonized or infected with CRE should be physically separated from non-colonized or non-infected patients using (a) single room isolation or (b) by cohorting patients with the same resistant pathogen.

\section{Recommendation 6}

Compliance with environmental cleaning protocols of the immediate surrounding area (that is, the "patient zone") of patients colonized or infected with CRE should be ensured.

\section{Recommendation 7}

Surveillance cultures of the environment for CRE should be considered when epidemiologically indicated.

\section{Recommendation 8}

Monitoring, auditing of the implementation of multimodal strategies and feedback of results to health care workers and decision-makers is recommended. 


\section{TREATMENT OPTIONS FOR INFECTIONS CAUSED BY CARBAPENEM RESISTANT PATHOGENS}

For treatment of infections caused by carbapenem resistant Gram-positive bacteria, there are some alternatives (such as glycopeptides, linezolid and daptomycin) to carbapenem. However, the therapeutic options are limited for infections caused by carbapenem resistant Gram-negative bacterial pathogens. ${ }^{4}$ Carbapenemase producing Gram-negative bacteria usually exhibit resistance to all or almost all beta-lactams and also commonly bear the genes encoding for resistance against fluoroquinolones and/or aminoglycosides. $^{4}$ Therefore, for these bacteria, the important last resort choices that are currently available include older agents such as polymyxins and fosfomycin, which were rarely used in the past because of efficacy and/or toxicity concerns, and the newer tigecycline. ${ }^{4}$ These drugs, based on the antimicrobial susceptibility data, can be used as: monotherapy using one of the possibly still active in vitro agents (which may be colistin, tigecycline or fosfomycin); or combination therapy without a carbapenem; or combination therapy with two or more drugs including at least one carbapenem, preferably when the carbapenem's MIC is 4 $\mathrm{mg} / \mathrm{L}{ }^{4}$ The last approach is considered as the most effective therapeutic strategy in terms of mortality. ${ }^{24}$

\section{Polymyxins}

Polymyxins are regarded as the most active agents against carbapenem resistant Gram-negative bacteria. ${ }^{25}$ They show their activity against most species in the family of Enterobacteriaceae (except for Serratia marcescens and Proteus, Providencia, Morganella, and Hafnia species), P. aeruginosa, A. baumannii, and some S. maltophilia strains. ${ }^{26}$

Chemically, they are cationic polypeptides. ${ }^{27}$ Polymyxin $\mathrm{B}$ and polymyxin $\mathrm{E}$ (colistin) are the two polymyxins available for clinical use. ${ }^{27}$ Polymyxin $\mathrm{B}$ is administered as its active form (polymyxin B sulfate) whereas colistin is administered as an inactive pro-drug, CMS (also called colistimethate) which results in a prolonged period of low plasma concentrations of the active drug and theoretically might increase the risk of resistance development. ${ }^{26,28}$

Polymyxins bind to negatively charged phosphate moieties in the lipid A fraction of lipopolysaccharide (LPS) present in the outer membrane of Gram-negative bacteria and causes the disruption of cell membranes that results in loss of intracellular products, thus achieving bactericidal activity. ${ }^{27}$

Polymyxin B was introduced into the clinical practice in the United States in the mid-1950s, and colistin towards the end of that decade. ${ }^{27}$ Due to toxicity (nephrotoxicity and neurotoxicity) concerns and availability of other antibacterials such as aminoglycosides and cephalosporins that were safer than polymyxins, they took as secondary place in antibiotic armamentarium. ${ }^{27}$ For decades, their use was confined to inhalation therapy in patients with cystic fibrosis, as a component of gastrointestinal tract decontamination regimens, and as topical antibiotic therapy. ${ }^{27}$

Polymyxins, due to their broad spectrum of activity, were "rediscovered" in the late 1990s, initially to address the therapeutic challenge posed by carbapenem-resistant $A$. baumannii (CRAB) and $P$. aeruginosa. ${ }^{27}$ Clinical studies have supported the effectiveness of polymyxins but reemphasized the concerns of nephrotoxicity and neurotoxicity associated with these drugs. ${ }^{29}$ Nephrotoxicity has been observed more frequently with colistin than polymyxin B. ${ }^{30}$ Though these drugs are useful, emergence of resistance has a negative impact on their continued utility for the treatment of CRE infections. ${ }^{27}$ Resistance has been reported from various parts of the world. ${ }^{31-33}$

\section{Fosfomycin}

Fosfomycin is a cell wall active agent discovered in 1969. ${ }^{27}$ It penetrates bacterial cell wall through the transport systems and inhibits the first step in the synthesis of peptidoglycan by blocking the formation of $\mathrm{N}$-acetylmuramic acid. ${ }^{27}$ It is a bactericidal drug with a broad spectrum of activity that includes Gram-positive (e.g. Enterococcus faecalis) and Gram-negative bacteria (e.g. Escherichia coli). ${ }^{34}$ Moreover, the drug has been found to be effective against approximately $80 \%$ of CRE, especially KPC-producing K. pneumoniae, even in strains that display decreased susceptibility to colistin and tigecycline. $^{35,36}$

The drug has a unique pharmacokinetic profile. ${ }^{27}$ The drug is $>50 \%$ bioavailable after oral administration, has a prolonged half-life, penetrates well into the tissues and is largely excreted unchanged into the urine, and achieves high penetration into the genitourinary system including the prostate. ${ }^{27}$ It is commonly used for treatment of bloodstream infection, endocarditis, meningitis, endophthalmitis, pneumonia, diabetic foot infections, peritonitis, gastroenteritis etc. It is well tolerated, with nausea, vomiting and diarrhea, or skin rashes, occurring in up to $5 \%$ of patients. ${ }^{37}$

Mechanism of action and safety profile of the drugs make it suitable for synergistic combination therapy, including regimens containing beta-lactams and aminoglycosides. ${ }^{27}$ When the drug is used alone, rapid emergence of resistance has been reported..$^{38}$

\section{Tigecycline}

Tigecycline is a glycylcycline bacteriostatic antibiotic, related to tetracyclines. ${ }^{27}$ It interferes with protein synthesis by binding to the $30 \mathrm{~S}$ ribosomal subunit. The 
drug received the first approval for clinical use from food and drug administration (FDA) in 2005. ${ }^{39}$

Tigecycline was designed to overcome tetracycline resistance mechanisms (ribosome protection and active efflux). ${ }^{40}$ It has broad spectrum of activity against both Gram positive and Gram negative pathogens, with notable exceptions of $P$. aeruginosa, Proteus species, and Providencia species. ${ }^{41}$ Among carbapenem-resistant Gram-negative pathogens, it shows its activity against the majority of CRE, A. baumannii, and S. maltophilia strains. Tigecycline is mostly used in combination regimens when treating carbapenem resistant Gramnegative infections. ${ }^{40}$

Since tigecycline is poorly absorbed through gastrointestinal tract, it requires intravenous administration. ${ }^{42}$ Adverse effects of this drug are usually mild and include nausea and vomiting. ${ }^{42}$

Tigecycline is primarily indicated for the treatment of complicated skin and soft tissue infections and complicated intra-abdominal infections when caused by CRE, among other MDR pathogens. ${ }^{43}$ Tigecycline is generally not recommended for bacteremia because of its bacteriostatic activity and low steady-state concentrations in serum at current dosing recommendation. ${ }^{40}$ However, it may still play an important role in the treatment of carbapenem-resistant $K$. pneumoniae bloodstream infection, especially as a part of combination regimens including colistin and carbapenems. ${ }^{27}$

Although tigecycline achieves low concentration in the urine, some studies have recommended its use in high doses for the treatment of urinary tract infection (UTI). ${ }^{44}$ In clinical practice, the use of tigecycline to treat CRE (especially KPC-producing $K$. pneumoniae) UTI is common, but may be associated with the development of resistance. $^{27}$

\section{Emerging drugs}

In the situation of evolving resistance against available antibiotics, world is currently in need of new antibiotics. A combination of avibactam and ceftazidime has been approved by FDA for treating complicated urinary tract and complicated intra-abdominal infections. ${ }^{45}$ Avibactam is a novel beta-lactamase inhibitor whereas ceftazidime is a third-generation cephalosporin. The combination shows in vitro activity against some CRE isolates. ${ }^{46}$ However, clinical data on its efficacy in severe infections caused by CRE are scarce.

Other novel antimicrobials which show in vitro activity against CRE are in different phases of clinical trials. ${ }^{45}$ Those antimicrobials include other combinations of avibactam with beta-lactams (i.e., ceftaroline fosamilavibactam and aztreonam-avibactam), the combinations of carbapenems with beta-lactamase inhibitors (i.e., meropenem-vaborbactam and imipenem/cilastatin- relebactam), a new aminoglycoside (plazomicin) and a new tetracycline (eravacycline).

\section{CONCLUSION}

Carbapenems are very useful drugs and are often regarded as the antibiotics of last resort in treatment of serious infections. However, emergence of resistance against this drug has posed a therapeutic challenge. Only few options are available for treatment of infections caused by carbapenem resistant pathogens.

Funding: No funding sources

Conflict of interest: None declared

Ethical approval: Not required

\section{REFERENCES}

1. Wallace PKM, Endimiani A, Taracila MA, Bonomo Ra. Carbapenems: past, present, and future. Antimicrob Agents Chemother. 2011;55:4943-60.

2. Bradley JS, Garau J, Lode H, Rolston KV, Wilson SE, Quinn JP. Carbapenems in clinical practice: a guide to their use in serious infection. Int $\mathbf{J}$ Antimicrob Agents. 1999;11:93-100.

3. World Health Organization (WHO). Guidelines for the prevention and control of carbapenem-resistant Enterobacteriaceae, Acinetobacter baumannii and Pseudomonas aeruginosa in health care facilities. WHO, November 2017. Available at: https://www.who.int/infection-prevention/ publications/guidelines-cre/en. Accessed 20 June 2020.

4. Meletis G. Carbapenem resistance: overview of the problem and future perspectives. Ther Adv Infect Dis. 2016;3(1):15-21.

5. Deck DH, Winston LG. Chapter 43: Beta-lactam and other cell wall membrane-active antibiotics. In: Katzung BG, editor. Basic and Clinical Pharmacology, 14e. New York, NY: McGraw-Hill; 2018. Available at: http://accessmedicine.mhmedical. $\mathrm{com} /$ content.aspx ?bookid $=2249 \&$ sectionid $=1752151$ 58. Accessed on 10 May 2020.

6. Bassetti M, Nicolini L, Esposito S, Righi E, Viscoli C. Current status of newer carbapenems. Curr Med Chem. 2009;16:564-75.

7. Gaibani P, Ambretti S, Berlingeri A, Gelsomino F, Bielli A, Landini MP, et al. Rapid increase of carbapenemase-producing Klebsiella pneumoniae strains in a large Italian hospital: surveillance period 1 March 30 September 2010. Euro Surveill. 2011;16(8):19800.

8. Nicasio AM, Kuti JL, Nicolau DP. The current state of multidrug-resistant Gram-negative bacilli in North America. Pharmacotherapy. 2008;28:235-49.

9. Patel G, Bonomo RA. Status report on carbapenemases: challenges and prospects. Expert Rev Anti Infect Ther. 2011;9:555-70. 
10. Sanchez M. Antibiotic resistance in the opportunistic pathogen Stenotrophomonas maltophilia. Front Microbiol. 2015;6:658.

11. Halat DM. Moubareck CA. The current burden of carbapenemases: Review of significant properties and dissemination among Gram-negative bacteria. Antibiotics. 2020;9:186.

12. Centers for Disease Control and Prevention (CDC). Health care associated infections: carbapenemresistant Enterobacteriaceae (CRE). Available at: https://www.cdc.gov/hai/organisms/cre/crepatients.html. Accessed on 20 June 2020.

13. Brink AJ. Epidemiology of carbapenem-resistant Gram-negative infections globally. Curr Opin Infect Dis. 2019;32:609-16.

14. World Health Organization (WHO). Prioritization of pathogens to guide discovery, research and development of new antibiotics for drug resistant bacterial infections, including tuberculosis. WHO, Sep 2017. Available at: https://www.who.int/ medicines/areas/rational_use/prioritization-ofpathogens/en. Accessed on 20 June 2020.

15. Shortridge D, Gales AC, Streit JM, Huband M, Tsakris A, Jones RN. Geographic and temporal patterns of antimicrobial resistance in Pseudomonas aeruginosa over 20 years from the SENTRY antimicrobial surveillance program. Open Forum Infect Dis. 2019;6(S1):63-8.

16. European Centre for Disease Prevention and Control. Surveillance of antimicrobial resistance in Europe. Annual report of the European Antimicrobial Resistance Surveillance Network (EARS Net) 2017. Available at: https://www.ecdc.europa.eu/sites/ default/files/documents/EARS-Net-report-2017update-jan-2019.pdf. Accessed on 20 June 2020.

17. Ruppe E, Woerther PL, Barbier F. Mechanisms of antimicrobial resistance in Gram-negative bacilli. Ann Intensive Care. 2015;5:21.

18. Eichenberger EM, Thaden JT. Epidemiology and mechanisms of resistance of extensively drug resistant Gram-negative bacteria. Antibiotics. 2019;8:37.

19. Weiner LM, Webb AK, Limbago B, Dedeck MA, Patel J, Kallen AJ, et al. Antimicrobial-resistant pathogens associated with healthcare-associated infections: summary of data reported to the National Healthcare Safety Network at the Centers for Disease Control and Prevention. Infect Control Hosp Epidemiol. 2016;37:1288-301.

20. Grundmann H, Glasner C, Albiger B, Aanensen DM, Tomlinson CT, Andrasevic, et al. Occurrence of carbapenemase producing Klebsiella pneumoniae and Escherichia coli in the European Survey of Carbapenemase-Producing Enterobacteriaceae (Eu SCAPE): a prospective, multinational study. Lancet Infect Dis. 2017;17:153-63.

21. Livorsi DJ, Chorazy ML, Schweizer ML, Balkenende EC, Blevins AE, Nair R, et al. A systematic review of the epidemiology of carbapenem-resistant
Enterobacteriaceae in the United States. Antimicrob Resist Infect Control. 2018;7:55.

22. McCann E, Srinivasan A, Ryke DA, Ye G, Pestel DD, Murray J, et al. Carbapenem-non susceptible Gram-negative pathogens in ICU and non-ICU settings in US hospitals in 2017: a multicenter study. Open Forum Infect Dis. 2018;5:1-7.

23. Kelly AM, Mathema B, Larson EL. Carbapenemresistant Enterobacteriaceae in the community: a scoping review. Int $\mathrm{J}$ Antimicrob Agents. 2017;50:127-34.

24. Tzouvelekis L, Markogiannakis A, Piperaki E, Souli M, Daikos GL. Treating infections caused by carbapenemase-producing Enterobacteriaceae. Clin Microbiol Infect. 2014;20:862-72.

25. Gales AC, Jones RN, Sader HS. Contemporary activity of colistin and polymyxin B against a worldwide collection of Gram-negative pathogens: results from the SENTRY Antimicrobial Surveillance Program (2006-09). J Antimicrob Chemother. 2011;66(9):2070-4.

26. Tran TB, Velkov T, Nation RL, Forrest A, Tsuji B, Bergen PJ, et al. Pharmacokinetics/ pharmacodynamics of colistin and polymyxin B: are we there yet? Int $\mathrm{J}$ Antimicrob Agents. 2016;48:592-7.

27. Perez F, Chakhtoura ENG, Wallace PK, Wilson BM, Bonomo RA. Treatment options for infections caused by carbapenem-resistant Enterobacteriaceae; can we apply "precision medicine" to antimicrobial chemotherapy? Expert Opin Pharmacother. 2016;17(6):761-81.

28. Zavascki AP, Bulitta JB, Landersdorfer CB. Combination therapy for carbapenem-resistant Gramnegative bacteria. Expert Rev Anti Infect Ther. 2013;11(12):1333-53.

29. Levin AS, Barone AA, Penco J, Santos MV, Marinho $\mathrm{S}$, Arruda EA, et al. Intravenous colistin as therapy for nosocomial infections caused by multidrugresistant Pseudomonas aeruginosa and Acinetobacter baumannii. Clin Infect Dis. 1999;28(5):1008-11.

30. Pike M, Saltiel E. Colistin and polymyxin-induced nephrotoxicity: focus on literature utilizing the RIFLE classification scheme of acute kidney injury. J Pharmacy Practice. 2014;27(6):554-61.

31. Mammina C, Bonura C, Bernardo DF, Aleo A, Fasciana T, Sodano C, et al. Ongoing spread of colistin-resistant Klebsiella pneumoniae in different wards of an acute general hospital, Italy, June to December 2011. Euro Surveill. 2012;17(33):20248.

32. Marchaim D, Chopra T, Pogue JM, Perez F, Hujer AM, Rudin S, et al. Outbreak of colistin-resistant, carbapenem-resistant Klebsiella pneumoniae in metropolitan Detroit, Michigan. Antimicrob Agents Chemother. 2011;55(2):593-9.

33. Kontopoulou K, Protonotariou E, Vasilakos K, Kriti M, Koteli A, Antoniadou E, et al. Hospital outbreak caused by Klebsiella pneumoniae producing KPC-2 beta-lactamase resistant to colistin. J Hosp Infect. 2010;76(1):70-3. 
34. Gobernado M. Fosfomycin. Rev Esp Quimioter. 2003;16(1):15-40.

35. Kaase M, Szabados F, Anders A, Gatermann SG. Fosfomycin susceptibility in carbapenem resistant Enterobacteriaceae from Germany. J Clin Microbiol. 2014;52(6):1893-7.

36. Endimiani A, Patel G, Hujer KM, Swaminathan M, Perez F, Rice LB, et al. In vitro activity of fosfomycin against Bla KPC-containing Klebsiella pneumoniae isolates, including those non susceptible to tigecycline and/or colistin. Antimicrob Agents Chemother. 2010;54(1):526-9.

37. Falagas ME, Giannopoulou KP, Kokolakis GN, Rafailidis PI. Fosfomycin: use beyond urinary tract and gastrointestinal infections. Clin Infect Dis. 2008;46(7):1069-77.

38. Fritzenwanker $M$, Imirzalioglu $C$, Herold $S$, Wagenlehner FM, Zimmer KP, Chakraborty $\mathrm{T}$. Treatment options for carbapenem resistant Gramnegative infections. Dtsch Arztebl Int. 2018;115:345-52.

39. Kasbekar N. Tigecycline: a new glycylcycline antimicrobial agent. Am J Health Syst Pharm. 2006;63:1235-43.

40. Doi Y. Treatment options for carbapenem-resistant Gram-negative bacterial infections. Clin Infect Dis. 2019;69(7):565-75.

41. Stein GE, Babinchak T. Tigecycline: an update. Diagn Microbiol Infect Dis. 2013;75:331-6.

42. Beauduy CE, Winston LG. Chapter 44: Tetracyclines, macrolides, clindamycin, chloramphenicol, streptogramins, and oxazolidinones. In: Katzung BG, editor. Basic and Clinical Pharmacology, 14e. New York, NY: McGraw-Hill; 2018. Available at: http://access medicine. mhmedical.com/content.aspx ?bookid $=2249$ \&sectionid=175215158. Accessed on 20 June 2020.

43. Bassetti M, Eckmann C, Bodmann KF, Dupont H, Heizmann WR, Montravers P, et al. Prescription behaviours for tigecycline in real-life clinical practice from five European observational studies. J Antimicrob Chemother. 2013;68(2):5-14.

44. Brust K, Evans A, Plemmons R. Tigecycline in treatment of multidrug-resistant Gram-negative bacillus urinary tract infections: a systematic review. J Antimicrob Chemother. 2014;69(10):2606-10.

45. Trecarichi EM, Tumbarello M. Therapeutic options for carbapenem-resistant Enterobacteriaceae infections. Virulence. 2017;8(4):470-84.

46. Falcone M, Paterson D. Spotlight on ceftazidime/avibactam: a new option for MDR Gramnegative infections. J Antimicrob Chemother. 2016;71(10):2713-22.

Cite this article as: Nepal HP, Paudel R. An overview of carbapenem, its resistance and therapeutic options for infections caused by carbapenem resistant bacteria. Int J Basic Clin Pharmacol 2020;9:1454-61. 